OPEN ACCESS

Edited by:

Milica S. Prostran,

University of Belgrade, Serbia

Reviewed by:

Nevena Divac,

University of Belgrade, Serbia

Scott Edward Counts,

Michigan State University,

United States

Dragan R. Milovanovic,

Faculty of Medical Sciences,

University of Kragujevac, Serbia

*Correspondence:

Efraim Jaul

jau@zahav.net.il;

efraimj@herzoghospital.org

Received: 03 August 2017 Accepted: 29 September 2017

Published: 17 October 2017

Citation:

Jaul E and Meiron O (2017) Systemic and Disease-Specific Risk Factors in

Vascular Dementia: Diagnosis and

Prevention.

Front. Aging Neurosci. 9:333. doi: 10.3389/fnagi.2017.00333

\section{Systemic and Disease-Specific Risk Factors in Vascular Dementia: Diagnosis and Prevention}

\author{
Efraim Jaul ${ }^{1 *}$ and Oded Meiron ${ }^{2}$ \\ 'Geriatric Skilled Nursing Department, Herzog Hospital, Hebrew University, Jerusalem, Israel, ${ }^{2}$ Clinical Research Center for \\ Brain Sciences, Herzog Hospital, Hebrew University, Jerusalem, Israel
}

In order to prevent the onset of vascular dementia $(\mathrm{VaD})$ in aging individuals, it is critical to detect clinically relevant vascular and systemic pathophysiological changes to signal the onset of its preceding prodromal stages. Identifying behavioral and neurobiological markers that are highly sensitive to $\mathrm{VaD}$ classification vs. other dementias is likely to assist in developing novel preventive treatment strategies that could delay the onset of disruptive psychomotor symptoms, decrease hospitalizations, and increase the quality of life in clinically-high-risk aging individuals. In light of empirical diagnostic and clinical findings associated with $\mathrm{VaD}$ pathophysiology, the current investigation will suggest a few clinically-validated biomarker measures of prodromal VaD cognitive impairments that are correlated with vascular symptomology, and VaD endophenotypes in non-demented aging people. In prodromal $\mathrm{VaD}$ individuals, distinguishing $\mathrm{VaD}$ from other dementias (e.g., Alzheimer's disease) could facilitate specific early preventive interventions that significantly delay more severe cognitive deterioration or indirectly suppress the onset of dementia with vascular etiology. Importantly, the authors conclude that primary prevention strategies should examine aging individuals by employing comprehensive geriatric assessment approach, taking into account their medical history, and longitudinally noting their vascular, systemic, cognitive, behavioral, and clinical functional status. Secondary prevention strategies may include monitoring chronic medication as well as promoting programs that facilitate social interaction and every-day activities.

\footnotetext{
Keywords: prodromal stage, vascular cognitive impairment, comprehensive geriatric assessment, cerebrovascular risk factors, prevention
}

\section{INTRODUCTION}

Vascular dementia $(\mathrm{VaD})$ is considered after Alzheimer's Disease (AD), as the most prevalent type of dementia (Lobo et al., 2000). According to the medical literature it is not clear how to distinguish between $\mathrm{AD}$ and $\mathrm{VaD}$ (Chui et al., 2000). More so, there is a lack of standardized medical evaluations and it is difficult to validate them in the clinical setting (Gorelick et al., 2011). Therefore, a few cardinal questions emerge regarding the detection and prevention of $\mathrm{VaD}$. First, is VaD a distinct clinical condition? Second, does VaD induce etiological precursors to AD pathophysiology? Accordingly, in the current theoretical investigation, we will suggest that $\mathrm{VaD}$ could lead to increased cerebrovascular impairments (e.g., ischemia) culminating in accelerated 
neurodegenerative processes. Alternatively, neurodegenerative processes initiate cerebrovascular pathologies (i.e., mixed dementia). Importantly, since both types of dementia are associated with cerebrovascular and cardiovascular conditions in the aging population, we raise a third fundamental question, can non-specific dementias represent a geriatric heterogenic syndrome related to a multi-factorial clinically-high-risk state culminating in the onset of $\mathrm{VaD}$ and $\mathrm{AD}$ such as in mixed dementia?

In order to explore these questions, we hypothesized that if the underlying pathophysiology is related to vascular conditions, prevention of abnormal vascular episodes (e.g., myocardial infarction, cerebrovascular accident) as well peripheral vascular disease, and promotion of healthy life styles (weight, diet, physical activity) will reduce the prevalence of cerebrovascular chronic pathologies, leading to lower prevalence of $\mathrm{VaD}$ (Christmas and Anderson, 2000). Accordingly, the authors aim to shed light on vascular conditions (hypertension, hyperlipidemia, hyper-coagulation, and arrhythmia), which represent the systemic pathological state that leads to vascular dementia, unlike AD-based primary pathophysiological processes that directly lead to specific neurodegenerative cellular processes (e.g., producing abnormal levels of tau proteins; Mattsson et al., 2009). Hence, we propose that VaD represents a geriatric syndrome, which could also indicate a clinically-highrisk state that increases the onset-probability of other dementias such as $\mathrm{AD}$ (and possibly frontotemporal dementia). In order to support this statement, we would like to discuss a few examples as observed in the clinical setting.

\section{CLINICAL PRESENTATION OF VASCULAR DEMENTIA}

In geriatric clinical evaluations, the most common method to distinguish $\mathrm{VaD}$ from $\mathrm{AD}$ requires identifying the location of the primary vascular abnormality in $\mathrm{VaD}$ vs. neurodegeneration (hippocampal atrophy) observed in $\mathrm{AD}$, usually at subcortical or cortical regions (Staekenborg et al., 2008). The cortical regions usually include damage to the larger blood vessels leading to lateralized sensorimotor symptoms. In contrast, the subcortical regions include small vessel thrombosis and embolism leading to brain parenchyma or chronic ongoing atherosclerosis. These subcortical vascular pathological conditions may negatively impact the deeper cerebral nuclei (e.g., basal ganglia nuclei) and white matter pathways (e.g., commissural/projection fibers). The clinical manifestations of these particular subcortical-vascular conditions might be subtle, including; focal motor signs (swallowing difficulties), gait disorder with instability and recurrent falls, urinary frequency/urgency, mild delirium (transient confusion), personality, and mood changes (depression, apathy, emotional fluctuations), and nondetectable mild cognitive impairments (MCIs) associated with accelerated decline in executive attention (Meiron et al., 2017). Mood changes and accelerated cognitive decline, could initially be difficult to detect in the prodromal pre-dementia stages, however, it is critical to detect these non-salient behavioral changes in order to signal the approaching onset of recurrent falls, delirium and swallowing difficulties, which could lead to increased hospitalizations and reduced quality of life. Identifying these early risk factors (i.e., accelerated cognitive decline between age 45-65), could direct family/geriatric clinicians to apply early preventive treatment strategies that will temporarily delay the onset of psychomotor symptoms (e.g., impaired spatial orientation, recurrent falling, and immobility), resulting in increased longevity, and sustain independent everydayfunctioning in elderly high-risk patients with VaD etiology. In many post-stroke patients, it is difficult to distinguish $\mathrm{VaD}$ onset from $\mathrm{AD}$ progression because the pre-stroke cognitive status was not noted. In addition, there are often sub-clinicalundetectable white matter lesions in post-stroke dementia patients. To increase the sensitivity for detecting and treating these pre-dementia conditions in pre or post-stroke patients, we recommend routine assessment of vascular and systemic risk factors to reduce susceptibility of $\mathrm{VaD}$ onset.

The main challenge is in detecting these geriatric clinical indications (Staekenborg et al., 2010). Unfortunately, many of the patients are usually referred to multiple specialized physicians (e.g., urologist, psychiatrist, neurologist) in response to the patients presenting symptoms, leading them to focus only on one component of the disease (depression, gait, instability, and urinary incontinence) instead of examining the entire physiological condition (medical history, full organs examination, blood test, vascular/brain imaging). In addition, geriatric vascular symptoms fluctuate according to the patient's daily activities/periods, and the effects of chronic drug treatment inadvertently lead clinicians toward misdiagnoses, which are likely to result in ineffective treatments. For example, due to the patient's bladder urgency the physician could prescribe antispasmodic drugs with anticholinergic action. However, the adverse effects of these drugs could be bladder retention and cognitive impairments which could deteriorate the patient's condition. If the same patient has other systemic risk factors that increase the frequency of recurrent falls, delirium and urinary infections the risk for development of $\mathrm{VaD}$ in later years significantly rises.

\section{SYSTEMIC AND SPECIFIC RISK FACTORS}

The main risk factors for $\mathrm{VaD}$ can be delineated by three prodromal or ongoing pathophysiological states; (1) Aging processes in the vessels (2) Cardio-vascular risk factors (3) cerebrovascular risk factors. Aging processes in vessels increases the risk for systemic pathophysiological processes (arteriosclerosis), and the risk for cerebrovascular damage. In relevance, atheroma deposits on the intimae of the arteries occlude the diameter of the lumen or disturb the blood flow. The thickness of the intima (due to proliferation of smooth muscle cells and connective tissue), leads to rigid and tortuous vessels. Pathological aging processes and cardio-vascular risk factors are also expressed by metabolic syndrome conditions; obesity (BMI $30 \mathrm{~kg} / \mathrm{m}^{2}$ or more), hypertension, insulin resistance, dyslipidemia, and increased waist circumference. One study 
reported that elderly people with cardiovaskular risk factor had hazard risk (HR) of 3.7 for developing dementia during the observational period (Solfrizzi et al., 2010). Another research found that MCI or Vascular Cognitive Impairments (VCI) are highly associated with VaD onset (Segura et al., 2009). Vascular brain damage due to increase cardiovascular risk factors can be characterized by two categories; clinical stroke (damage of large arteries) or subclinical "lacunar" or "silent" brain infraction (injury of small arteries). After clinically manifested stroke, dementia or cognitive impairment develops in $6-32 \%$ patients during the follow up period of 3 months to 20 years (Ivan et al., 2004; Rist et al., 2013). In a cohort-study of patients after lacunar stroke, related to small vessels diseases, $50 \%$ of the patients suffered later from MCI or dementia (Jacova et al., 2012). In a systematic review and meta-analysis of 73 papers published between the years of 1950 and 2009 examining pre-stroke and post-stroke dementia patients, the rate of dementia onset ranged between 7.4 and $41.3 \%$ (Pendlebury et al., 2009). After adjustment of the methods and mix cases, findings indicated that $10 \%$ of the patients with first stroke had pre-stroke dementia, and $10 \%$ of the patients subsequently developed dementia, while more than $33 \%$ of the patients developed dementia after a recurrent stroke (Pendlebury et al., 2009). These finding emphasizes the important role of secondary prevention (e.g., statin prescription for lowering cholesterol and triglycerides, antiplatelet drug and anticoagulant use in order to prevent thromboembolism), immediately after the first stroke. Systemic factors such as high serum levels of homocysteine or LDL-lipoproteins, hypercoagulability conditions, diabetes mellitus with inadequate glycemia control, smoking, and obesity should be examined and treated within primary and secondary prevention strategies.

\section{EARLY DETECTION AND PREVENTION OF VASCULAR DEMENTIA}

In attempt to support our theoretical premise it is prudent to further examine our theoretical model by asking "why is it clinically relevant to distinguish VaD from other dementias?" The main clinical benefit in identifying non-demented prodromal $\mathrm{VaD}$ cases vs. non-demented prodromal $\mathrm{AD}$ cases (such as detecting subtle age-related vascular vulnerabilities by utilizing known disease-specific endophenotypes), could lead to early preventive interventions in a critical time point preceding the pathophysiological phenotypical expression of $\mathrm{VaD}$. Endophenotypes are physiological markers (e.g., hippocampal atrophy in individuals with Alzheimer's disease) in humans that are genetically correlated with disease liability (Glahn et al., 2007). Endophenotypes are disease-specific biomarkers that are generally closer to the action of the gene, and thus exhibit higher genetic-risk sensitivity for specific neurological disorders (Glahn et al., 2007). Suppose, for example, that a specific VaD endophenotype is discovered and that the association of that endophenotype with white matter lesions observed in $\mathrm{VaD}$ prior to phenotypical expression (such as mild cognitive impairment ordelirium) is proved. The finding could pawed the way for subsequent researches for novel medication which modulate that endophenotype. In the future, evidence-based treatment strategies which prevent cerebral ischemic episodes as well as the consequential onset of dementia could be finally established. Some researchers proposed the abnormal expression of myelin basic protein (MBP) to be the endophenotype for development of $\mathrm{VaD}$ but further validation studies were needed before definite proof of this concept (Bjerke et al., 2011; Meiron et al., 2017). Other research groups favored the assessment of total tau protein as a specific marker for $\mathrm{AD}$. It has been observed that total tau protein levels in the CSF were significantly increased in $\mathrm{AD}$ vs. both $\mathrm{VaD}$ and healthy controls (Bjerke et al., 2011). In conclusion, the markers of prodromal subtle-phase that precedes $\mathrm{VaD}$ promised an era of emerging preventive and therapeutic strategies which could suppress or delay the onset of debilitating dementia cognitive-symptoms, as well as altering neurodegenerative disease progression.

On a cognitive level, high-risk elderly pre-dementia individuals may exhibit VCI (O’Brien et al., 2003), or nondemented MCI, or non-salient accelerated cognitive decline (pre-MCI state that is less severe in comparison to amnesic or progressive $\mathrm{MCI}$ ), therefore it is important to detect significantly lower verbal memory scores in MCI vs. non-MCI controls using standard cognitive testing measures such as the Rey Auditory Verbal Learning Test (RAVLT) and the California Verbal Learning Test (Deiber et al., 2009; Albert et al., 2011). In most cases, verbal memory is the most prominent cognitive-domain that gradually deteriorates and this pre-condition is likely to progress to $\mathrm{AD}$ in $12 \%$ of $\mathrm{MCI}$ patients within a consecutive 24-month period or between 40 and $60 \%$ cumulatively, over a life-time period (Hua et al., 2008; Babiloni et al., 2010). However, in prodromal $\mathrm{VaD}$, the impact of MCI symptoms on daily activities is less apparent (particularly to the family and immediate community) than subtle gait impairments leading to increased risk of falling (Jokinen et al., 2006). Therefore, it is clinically essential to identify early high-risk MCI cases with vascular history before they exhibit significant cognitive impairments that lead to an irreversible physical condition due to spatial disorientation or falling. In support, early detection of increased dual-task (e.g., walking while performing a cognitive task) deficits in elderly controls (Manor et al., 2016) could be perceived as an early $\mathrm{VaD}$ phenotypical expression preceding gait problems and recurrent falls, particularly if the person has cardiovascular history. As these types of dual-task assessments are associated with left prefrontal cortex excitability as well as with executive attention tasks (Meiron et al., 2013) that are associated with prefrontal-cortex functional connectivity (Meiron and Lavidor, 2014), we can employ these "prefrontal activity" neuro-cognitive testing procedures to detect accelerated pathological cognitive decline. In support, progressive MCI patients display significantly reduced prefrontal theta EEG activity (during the onset of a working memory trial requiring the correct rejection of a non-target stimulus) vs. stable MCI, and vs. healthy controls. Hence, if an elderly person without any memory complaints begins to show clinically significant deterioration in dual-task scores (Manor et al., 2016), as well as attaining a verbal memory score (e.g., Rey Auditory Verbal 
Learning Test) that is at least $1.5 S D$ lower than the age-matched group-mean (Albert et al., 2011), we suggest that it would be advisable to proceed with functional EEG imaging to exclude or identify progressive MCI (Deiber et al., 2009). Additionally, clinical assessment should include the Petersen MCI criteria, wherein single domain amnestic MCI will be seen more as a predictor for $\mathrm{AD}$ and/multi-domain $\mathrm{MCI}$ may be more indicative of underlying vascular pathology (Albert et al., 2011). Finally, in order to rule out $\mathrm{VaD}$ diagnosis, we suggest that white matter lesions should be examined (associated with ineffective cerebral connectivity) along with hippocampal-atrophy, and both conditions should be excluded (Hua et al., 2008; Cao et al., 2010; Ardekani et al., 2014). All these procedures (MRI, EEG, Hachinski Ischemic/Ischemia Scores/Scale) are available and are non-invasive, facilitating the cooperation of more prospective elderly patients willing to be examined and clarifying the clinical picture for the treating physicians. In support, the Hachinski Ischemic Scores can be applied routinely to reliably distinguish vascular dementia from other dementias (Moroney et al., 1997).

\section{DISCUSSION}

In response to the diagnostic and clinical implications for early detection of prodromal $\mathrm{VaD}$, our theoretical perspective suggests that $\mathrm{VaD}$ represents a final pathway induced by a multi factorial pathophysiological (e.g., risk factors such as lacunar stroke and atherosclerosis) state leading to a geriatric syndrome. Primary VaD prevention strategies should focus on modifying daily life styles such as: smoking, caloric intake, carbohydrates and salt in diet, aerobic and anaerobic physical exercise. Secondary prevention strategies may include monitoring of antilipemics, antidiabetics, antihypertensives, antiplatelets and anticoagulants medications, and tertiary prevention measures such as rehabilitation programs after stroke as well as promoting programs that facilitate social interaction and every day independent activities are also important.

In elderly person, we have to allow sufficient blood and oxygen perfusion to, and in the brain, hence, it is advisable to be cautious with aggressive hypo-perfusion medications, which lower blood pressure and glucose levels in the brain. To accomplish an effective holistic approach in preventing or treating $\mathrm{MCI} / \mathrm{VaD}$ it is critical that the family physician coordinates the treatment with other expert-clinicians (e.g., social worker, occupational therapist, neuropsychologist) in order to advance a multidisciplinary prevention-treatment to delay the onset of dementia and related functional impairments.

\section{REFERENCES}

Albert, M. S., DeKosky, S. T., Dickson, D., Dubois, B., Feldman, H. H., Fox, N. C., et al. (2011). The diagnosis of MCI due to Alzheimer's disease: recommendations from the national institute on aging-Alzheimer's association workgroups on diagnostic guidelines for Alzheimer's disease. Alzheimers Dement. 7, 270-279. doi: 10.1016/j.jalz.2011. 03.008

American Psychiatric Association (2013). Diagnostic and Statistical Manual of Mental Disorders, Fifth Edition (DSM5). Arlington, VA: American Psychiatric Association.
Particularly in its prodromal early stages, undiagnosed VaD progression in geriatric populations stems from the lack of disease-specific criteria to identify $\mathrm{VaD}$ as a distinct clinical entity. Does this imply a clinical need for an appropriate new diagnostic entity associated more with VCI (O’Brien et al., 2003). VCI can result from multiple pathophysiological pathways. For instance, post-stroke patients display focal cognitive deficits related to the area of infraction or/and suffer from hypo-perfusion affecting deeper neural structures (associated with global cognitive impairments) indicating a systemic cerebrovascular atherosclerosis. VCI can be detected in patients suffering from a manifested and subclinical stroke, silent white matter lesions and arrhythmias (e.g., atrial fibrillation). Systemic chronic diseases significantly promote the pathogenesis of blood-vessel diseases. Sporadic VCI evolution to MCI (i.e., mild neurocognitive disorder; American Psychiatric Association, 2013) may represent a shift from mild cognitive symptoms to a deterioration of functional capacity status (e.g., ADL and cognitive impairments) that is diagnosed as mixed dementia, associated with $\mathrm{AD}$ and $\mathrm{VaD}$ debilitating cognitive impairments.

\section{CONCLUSION}

The authors suggest that the optimal prevention-treatment approaches to reduce $\mathrm{VaD}$ prevalence or severity require early comprehensive geriatric assessment. The term "comprehensive" indicates taking into account at the overall clinical features and not just the presenting symptoms (gait, urinary urgency, depression). For instance, Hachinski Ischemic Scores (HIS $\geq$ 7) are considered a sensitive and specific diagnostic marker for identifying $\mathrm{VaD}$ with high inter-rater reliability (Moroney et al., 1997). Thus, HIS scores in combination with neuroimaging, as well as vascular pathology imaging (e.g., pulsed arterial spin labeling, pASL; Cantin et al., 2011) could reliably indicate $\mathrm{VaD}$ pathophysiology. To accomplish an effective multifactorial approach in identifying or preventing $\mathrm{VIC} / \mathrm{VaD}$ onset it is critical to apply early multidisciplinary preventiontreatments utilizing systemic-vascular-neurocognitive quantifiable cross-validated markers to delay or possibly prevent the onset of dementia and related functional impairments.

\section{AUTHOR CONTRIBUTIONS}

Both authors contributed equally to the conception and composition of this paper.

Ardekani, B. A., Bachman, A. H., Figarsky, K., and Sidtis, J. J., (2014). Corpus callosum shape changes in early Alzheimer's disease: an MRI study using the OASIS brain database. Brain Struct. Funct. 219, 343-352. doi: 10.1007/s00429-013-0503-0

Babiloni, C., Frisoni, G. B., Vecchio, F., Pievani, M., Geroldi, C., De Carli, C., et al. (2010). Global functional coupling of resting EEG rhythms is related to white-matter lesions along the cholinergic tracts in subjects with amnesic mild cognitive impairment. J. Alzheimers Dis. 19, 859-871. doi: 10.3233/JAD-2010-1290

Bjerke, M., Zetterberg, H., Edman, Å., Blennow, K., Wallin, A., and Andreasson, U. (2011). Cerebrospinal fluid matrix metalloproteinases and tissue inhibitor 
of metalloproteinases in combination with subcortical and cortical biomarkers in vascular dementia and Alzheimer's disease. J. Alzheimers Dis. 27, 665-676. doi: 10.3233/JAD-2011-110566

Cantin, S., Villien, M., Moreaud, O., Tropres, I., Keignart, S., and Chipon, A., et al (2011). Impaired cerebral vasoreactivity to CO2 in Alzheimer's disease using BOLD fMRI. Neuroimage 58, 579-587. doi: 10.1016/j.neuroimage.2011.06.070

Cao, X., Guo, Q., Zhao, Q., Jin, L., Fu, J., and Hong, Z., (2010). The neuropsychological characteristics and regional cerebral blood flow of vascular cognitive impairment-no dementia. Int. J. Geriatr. Psychiatry 25, 1168-1176. doi: $10.1002 /$ gps.2458

Christmas, C., and Anderson, R. A. (2000). Exercise and older patients: guide-line for the clinician. J. Am. Geriat. Soc. 48, 318-324. doi: 10.1111/j.1532-5415.2000.tb02654.x

Chui, H. C., Mack, W., Jackson, J. E., Mungas, D., Reed, B. R., Tinklenberg, J., et al. (2000). Clinical criteria for the diagnosis of vascular dementia: a multicenter study of comparability and interrater reliability. Arch. Neurol. 57, 191-196. doi: 10.1001/archneur.57.2.191

Deiber, M. P., Ibañez, V., Missonnier, P., Herrmann, F., Fazio-Costa, L., Gold, G., et al. (2009). Abnormal-induced theta activity supports early directedattention network deficits in progressive MCI. Neurobiol. Aging 30, 1444-1452. doi: 10.1016/j.neurobiolaging.2007.11.021

Glahn, D. C., Thompson, P. M., and Blangero, J. (2007). Neuroimaging endophenotypes: strategies for finding genes influencing brain structure and function. Hum. Brain Mapp. 28, 488-501. doi: 10.1002/hbm.20401

Gorelick, P. B., Scuteri, A., Black, S. E., DeCarli, C., Greenberg, S. M., Iadecola, C., et al. (2011). Vascular contribution to cognitive impairment and dementia. A statement for health care professionals from the american heart and stoke association. Stroke 42, 2672-2713. doi: 10.1161/STR.0b013e3182299496

Hua, X., Leow, A. D., Lee, S., Klunder, A. D., Toga, A. W., Lepore, N., et al. (2008). 3D characterization of brain atrophy in Alzheimer's disease and mild cognitive impairment using tensor-based morphometry. Neuroimage 41, 19-34. doi: 10.1016/j.neuroimage.2008.02.010

Ivan, C. S., Seshadri, S., Beiser, A., Au, R., Kase, C. S., Kelly-Hayes, M., et al. (2004). Dementia after stroke: the framingham study. Stroke 35, 1264-1268. doi: 10.1161/01.STR.0000127810.92616.78

Jacova, C., Pearce, L. A., Costello, R., McClure, L. A., Holliday, S. L., Hart, R. G., et al. (2012). Cognitive impairment in lacunar strokes: the SPS3 trial. Ann. Neurol. 72, 351-362. doi: 10.1002/ana.23733

Jokinen, H., Kalska, H., Mäntylä, R., Pohjasvaara, T., Ylikoski, R., Hietanen, M., et al. (2006). Cognitive profile of subcortical ischemic vascular disease. J. Neurol. Neurosurg. Psychiatr. 77, 28-33. doi: 10.1136/jnnp.2005.069120

Lobo, A., Launer, L. J., Fratiglioni, L., Andersen, K., Di Carlo, A., Breteler, M. M., et al. (2000). Prevalence of dementia and major subtypes in Europe: a collaborative study of population-based cohorts. Neurologic diseases in the elderly research group. Neurology 54, S4-S9. Available online at: http://ovidsp. tx.ovid.com/sp-3.16.0b/ovidweb.cgi

Manor, B., Zhou, J., Jor'dan, A., Zhang, J., Fang, J., and Pascual-Leone, A. (2016). Reduction of dual-task costs by noninvasive modulation of prefrontal activity in healthy elders. J. Cogn. Neurosci. 28, 275-281. doi: 10.1162/jocn_a_00897

Mattsson, N., Zetterberg, H., Hansson, O., Andreasen, N., Parnetti, L., Jonsson, M., et al. (2009). CSF biomarkers and incipient Alzheimer disease in patients with mild cognitive impairment. JAMA 302, 385-393. doi: 10.1001/jama.2009.1064

Meiron, O., and Lavidor, M. (2014). Prefrontal oscillatory stimulation modulates access to cognitive control references in retrospective metacognitive commentary. Clin. Neurophysiol. 125, 77-82. doi: 10.1016/j.clinph.2013.06.013
Meiron, O., Hermesh, H., Katz, N., and Weizman, A. (2013). Executive attention deficits in schizophrenia: putative mandatory and differential cognitive pathology domains in medicated schizophrenia patients. Psychiatry Res. 209, 1-8. doi: 10.1016/j.psychres.2012.09.057

Meiron, O., Rosset, E., and Braw, Y. (2017). "Putative neurocognitive marker for early non-salient accelerated cognitive decline in older adults," in Horizons in Neuroscience Research, Vol. 26, eds A. Costa and E. Villalba (New York, NY: Nova Science Publishers), 93-100.

Moroney, J. T., Bagiella, E., Desmond, D. W., Hachinski, V. C., Mölsä, P. K., Gustafson, L., et al (1997). Meta-analysis of the Hachinski ischemic score in pathologically verified dementias. Neurology 49, 1096-1105. doi: 10.1212/WNL.49.4.1096

O’Brien, J. T., Erkinjuntti, T., Reisberg, B., Roman, G., Sawada, T., Pantoni, L., et al. (2003). Vascular cognitive impairment. Lancet Neurol. 2, 89-98. doi: 10.1016/S1474-4422(03)00305-3

Pendlebury, S. T., Rothwell, P. M., Snaphaan, L., and de Leeuw, F. E. (2009). Prevalence, incidence, and factors associated with pre-stroke and post-stroke dementia: a systematic review and meta-analysis. Lancet Neurol. 8, 1006-1018. doi: 10.1016/S1474-4422(09)70236-4

Rist, P. M., Chalmers, J., Arima, H., Anderson, C., Macmahon, S., Woodward, M., et al. (2013). Baseline cognitive function, recurrent stroke, and risk of dementia in patients with stroke. Stroke 44, 1790-1795. doi: 10.1161/STROKEAHA.111.680728

Segura, B., Jurado, M. A., Freixenet, N., Albuin, C., Muniesa, J., and Junqué, C. (2009). Mental slowness and executive dysfunctions in patients with metabolic syndrome. Neurosci. Lett. 462, 49-53. doi: 10.1016/j.neulet.2009. 06.071

Solfrizzi, V., Scafato, E., Capurso, C., D’Introno, A., Colacicco, A. M., Frisardi, V., et al. (2010). Metabolic syndrome and the risk of vascular dementia: the Italian longitudinal study on ageing. J. Neurol. Neurosurg. Psychiatr. 81, 433-440. doi: 10.1136/jnnp.2009.181743

Staekenborg, S. S., Su, T., van Straaten, E. C., Lane, R., Scheltensm, P., and Barkhof, F. (2010). Behavioral and psychological symptoms in vascular dementia; differences between small- and large-vessel disease. J. Neurol. Neurosurg. Psychiatr. 81, 547-552. doi: 10.1136/jnnp.2009. 187500

Staekenborg, S. S., van der Flier, W. M., van Straaten, E. C., Lane, R., Barkhof, F., and Scheltens, P. (2008). Neurological signal relation to type of cerebrovascular disease in vascular dementia. Stroke 39, 317-322. doi: 10.1161/STROKEAHA.107.493353

Conflict of Interest Statement: The authors declare that the research was conducted in the absence of any commercial or financial relationships that could be construed as a potential conflict of interest.

The reviewer ND and handling Editor declared their shared affiliation, and the handling Editor states that the process nevertheless met the standards of a fair and objective review

Copyright (c) 2017 Jaul and Meiron. This is an open-access article distributed under the terms of the Creative Commons Attribution License (CC BY). The use, distribution or reproduction in other forums is permitted, provided the original author(s) or licensor are credited and that the original publication in this journal is cited, in accordance with accepted academic practice. No use, distribution or reproduction is permitted which does not comply with these terms. 